\title{
THE GROWTH OF \\ THE EXPECTED NUMBER OF REAL ZEROS OF A RANDOM POLYNOMIAL
}

\author{
RICHARD GLENDINNING
}

(Received 27 January 1987; revised 8 July 1987)

Communicated by T. C. Brown

\begin{abstract}
Let $X_{0}, X_{1}, \ldots, X_{n}, \ldots$ be a stationary Gaussian process. We give sufficient conditions for the expected number of real zeros of the polynomial $Q_{n}(z)=\sum_{j=0}^{n} X_{j} z^{j}$ to be $(2 / \pi) \log n$ as $n$ tends to infinity.

1980 Mathematics subject classification (Amer. Math. Soc.) (1985 Revision): primary 60 H 25; secondary 60 G 17.

Keywords and phrases: real zeros, random polynomials, asymptotic growth, stationary Gaussian process, spectral density, uniformly mixing, Kac-Rice formula, Toeplitz forms.
\end{abstract}

\section{Introduction}

Let $\left(X_{j}, j=0,1, \ldots\right)$ be a sequence of real-valued random variables satisfying the following moment conditions.

$$
E\left(X_{j}\right)=0 \text { and } E\left(X_{j}\right)^{2}=1 \text { for all } j \geq 0 .
$$

A random polynomial of order $n$ is given by

$$
Q_{n}(z)=\sum_{j=0}^{n} X_{j} z^{j}, \quad z \in Z .
$$

(C) 1989 Australian Mathematical Society $0263-6115 / 89 \$ A 2.00+0.00$ 
Denote the number of real zeros of (1.2) in the interval $(a, b)$ by $N_{n}((a, b))$. Ibragimov and Maslova (1971a) show that

$$
E N_{n}((-\infty, \infty)) \sim(2 / \pi) \log n \text { as } n \rightarrow \infty,
$$

when $\left(X_{j}, j=0,1, \ldots\right)$ is a sequence of independent and identically distributed random variables satisfying (1.1) and belonging to the domain of attraction of the normal law.

In this paper we give sufficient conditions for (1.3) to hold when $\left(X_{j}\right.$, $j=0,1, \ldots)$ is a stationary real-valued Gaussian process satisfying (1.1).

The earliest work on polynomials with dependent coefficients is due to Hamblen (1956) who examined the expected number of real zeros of $X_{0}+X_{1} z+z^{2}$ when $\left(X_{0}, X_{1}\right)$ is a bivariate normal random vector satisfying (1.1) for $j=0,1$.

Much subsequent work, Sambandham $(1977,1979)$, has concentrated on the case where $\left(X_{j}, j=0,1, \ldots, n\right)$ is a multivariate normal random vector with simple correlation matrix. Shenker (1981) gives two sets of sufficient conditions for (1.3) to hold when $\left(X_{j}, j=0,1, \ldots\right)$ is a stationary real-valued Gaussian process satisfying (1.1).

We extend Theorem 1 of Shenker (1981) and give some complementary results. We also consider the case where $\left(X_{j}\right)$ is a stationary uniformly mixing Gaussian process. In contrast to Shenker our conditions are in terms of the spectral density associated with $\left(X_{j}\right)$ rather than its autocorrelation function.

The analogous problem for random trigonometric polynomials has been studied in Sambandham (1976a, 1976b), Sambandham and Maruthachalam (1978), Renganathan and Sambandham (1984) and Bharucha-Reid and Sambandham (1986).

The main results of this paper are given in Section 2. The key step in our proof is to estimate $E N_{n}([0,1])$ by counting the sign changes of a piece-wise approximation to $Q_{n}(x)$. This approach has been used by Ibragimov and Maslova (1971a, 1971b) to derive sufficient conditions for (1.3) in the iid case. Note that this extension depends on the fact that $Q_{n}(x)$ behaves like a polynomial with Gaussian coefficients for large $n$. Our results are compared with earlier work in Section 7.

\section{Main results}

Theorems 2.1, 2.2 and 2.3 give sufficient conditions for $E N_{n}([0,1]) \sim(1 / 2 \pi)$ $\log n$ as $n$ tends to infinity. Sufficient conditions for (1.3) follow when these conditions are also satisfied by the sequence $\left((-1)^{j} X_{j}, j \geq 0\right)$. 
First we give some notation. Let $r_{j}=E\left(X_{0} X_{j}\right)$. The spectral density associated with $\left(r_{j}, j \geq 0\right)$ is denoted by $f(\theta)$. So $r_{v}=\int_{-\pi}^{\pi} e^{-i v \theta} f(\theta) d \theta$ for $v \geq 0$.

THEOREM 2.1. Let $\left(X_{j}, j \geq 0\right)$ be a stationary real-valued Gaussian process satisfying (1.1). Suppose that

(i) there is an interval $[-b, b], 0<b \leq \pi$, where $f(\theta)$ can be uniformly approximated by the partial sums, $S_{n} f(\theta)$ of its Fourier series development, and

(ii) $\infty>M \geq f(\theta) \geq m>0, \theta \in[-\pi, \pi]$.

Then

$$
E N_{n}([0,1]) \sim(1 / 2 \pi) \log n, \quad n \rightarrow \infty
$$

THEOREM 2.2. The conclusions of Theorem 2.1 hold if condition (i) is satisfied with $b=\pi$ and there is a constant $\alpha$ such that

$$
\frac{1}{2}+\sum_{j=1}^{n} r_{j} x^{j} \geq \alpha>0, \quad x \in[0,1], n \geq N_{0},
$$

for some integer $N_{0}$.

Let $M_{a}^{b}$ be the $\sigma$-algebra generated by the random variables $\left(X_{j}, j=a, \ldots, b\right)$. Then $\left(X_{j}, j \geq 0\right)$ is said to be uniformly mixing if there exists a non-increasing sequence $(\phi(k), k \geq 1)$ such that

$$
\sup |P(A B)-P(A) P(B)| \leq \phi(k) P(A), \quad \phi(k) \downarrow 0,
$$

where the supremum in (2.1) is taken over all $A \in M_{0}^{n}$ and $B \in M_{n+k}^{\infty}, n \geq 0$.

THEOREM 2.3. When $\left(X_{j}\right)$ is a uniformly mixing Gaussian process with (i) $\sum_{k=1}^{\infty} \phi^{1 / 2}(k)<\infty$ and (ii) $f(0)>0$, then the conclusions of Theorem 2.1 hold.

When the conditions given in Theorems $2.1,2.2$ and 2.3 are also satisfied by the sequence $\left((-1)^{j} X_{j}, j \geq 0\right)$ we have (1.3). In particular we have the following result.

COROLLARY 2.1. If the conditions of Theorem 2.1 hold with $b=\pi$ then (1.3) follows.

We give a brief overview of the proof of these results. We partition $[0,1]$ into intervals $I_{n}^{1}, I_{n}^{2}$ and $I_{n}^{3}$ so that

$$
E N_{n}\left(I_{n}^{1}\right)+E N_{n}\left(I_{n}^{3}\right)=o(\log n) \text { and } E N_{n}\left(I_{n}^{2}\right) \sim(1 / 2 \pi) \log n
$$


as $n$ tends to infinity. These quantities depend on certain variance and covariance estimates derived in Section 3. Let $\hat{N}_{n}(a, b)$ be the number of sign changes of a piece-wise linear approximation to $Q_{n}(x)$. In Section 4 we estimate $\mid E \hat{N}_{n}(a, b)-$ $E N_{n}([a, b]) \mid$. In Section 5 we construct a sequence $\left(x_{j}, j=p_{0}^{n}, \ldots, p_{1}^{n}\right)$ and estimate $E N_{n}\left(I_{n}^{2}\right)$ by the sum $\sum_{j=p_{0}^{n}}^{p_{1}^{n}-1} E \hat{N}_{n}\left(x_{j}, x_{j+1}\right)$. In Section 6 we show that $E N_{n}\left(I_{n}^{1}\right)$ and $E N_{n}\left(I_{n}^{3}\right)$ are $o(\log n)$ as $n$ tends to infinity.

Partition $[0,1]$ into intervals $I_{n}^{1}, I_{n}^{2}$ and $I_{n}^{3}$ given by

$$
\begin{aligned}
& I_{n}^{1}=\left[0,1-(\log n)^{-1 / 2}\right], \\
& I_{n}^{2}=\left[1-(\log n)^{-1 / 2}, 1-n^{-1} \log \log n\right], \\
& I_{n}^{3}=\left[1-n^{-1} \log \log n, 1\right] \quad \text { for } n \geq 3 .
\end{aligned}
$$

Throughout this paper we take $C$ to be a generic positive constant.

\section{Covariance estimates}

Let $k_{n}(x, y)=E Q_{n}(x) Q_{n}(y)$ and $r_{n}(x, y)=\operatorname{Cor}\left(Q_{n}(x), Q_{n}(y)\right)$. We estimate $k_{n}(x, y)$ and $r_{n}(x, y)$ for $x, y$ satisfying certain conditions. For $x \in I_{n}^{3}$ we derive upper and lower bounds for $k_{n}(x, x)$. We weaken the conditions of a result due to Yoshihara (1978).

In this section we take $\left(X_{j}, j \geq 0\right)$ to be a sequence of not necessarily Gaussian random variables satisfying (1.1). Let $r_{j}=E X_{0} X_{j}$. We denote the spectral density associated with $\left(r_{j}, j \geq 0\right)$ by $f(\theta)$. Where convenient we interpret $f(\theta)$ as a function defined on the set of complex numbers with modulus one. So $f\left(e^{i \theta}\right)$ and $f(\theta), \theta \in[-\pi, \pi]$, are equivalent.

Let $T_{n}(\theta)$ be a trigonometric polynomial of order $n$ with real coefficients. Suppose that

$$
T_{n}(\theta)=\sum_{v=-n}^{n} c_{v} e^{i v \theta}, \quad \theta \in[-\pi, \pi], c_{v} \in R
$$

Define

$$
G\left(T_{n}, x\right)=2 \pi\left(\sum_{v=0}^{n} c_{v} x^{v}-c_{0} / 2\right)
$$

Then

$$
\begin{aligned}
k^{a}\left(T_{n}, x, y\right) & =\frac{G\left(T_{n}, x\right)+G\left(T_{n}, y\right)}{1-x y}, \quad x, y \in[0,1), \\
r^{\prime}(x, y) & =\frac{\left(1-x^{2}\right)^{1 / 2}\left(1-y^{2}\right)^{1 / 2}}{1-x y}, \quad x, y \in[0,1) .
\end{aligned}
$$

Take $\infty>d>0$. We estimate $k_{n}(x, y)$ for $x, y$ satisfying the conditions $r^{\prime}(x, y) \geq$ $d>0$ and $x, y \in I_{n}^{2}$. 
THEOREM 3.1. Suppose that $0<b \leq \pi$. If

(i) $f(\theta)$ can be uniformly approximated by the partial sums of its Fourier series development, $S_{n} f(\theta)$ for $\theta \in[-b, b]$,

(ii) $f(\theta) \leq A<\infty, \theta \in[-\pi, \pi]$,

(iii) $f(0)>0$,

then

$$
\sup _{\substack{x, y \in I_{n}^{2} \\ \Gamma^{\prime}(x, y) \geq d>0}}\left|\frac{k_{n}(x, y)}{k^{a}\left(S_{n} f, x, y\right)}-1\right| \rightarrow 0 \quad \text { as } n \rightarrow \infty .
$$

COROLLARY 3.1. Under the conditions of the previous theorem we have

$$
\sup _{\substack{x, y \in I_{n}^{2} \\ r^{\prime}(x, y) \geq d>0}}\left|r_{n}(x, y)-r^{\prime}(x, y)\right| \rightarrow 0 \text { as } n \rightarrow \infty .
$$

For $x, y \in I_{n}^{1} \cup I_{n}^{2}$ we have

THEOREM 3.2. Suppose that condition (i) in Theorem 3.1 is satisfied with $b=\pi$. If there is a constant $\alpha$ and an integer $N_{0}$ such that

(ii) $G\left(S_{n} f, x\right) \geq \alpha>0$ for $n \geq N_{0}, x \in[0,1]$, then the conclusions of Theorem 3.1 hold for $x, y \in I_{n}^{1} \cup I_{n}^{2}$. Next we give a bound for the variance of $Q_{n}(x)$ for $x \in I_{n}^{2} \cup I_{n}^{3}$.

THEOREM 3.3. Suppose that conditions (ii) and (iii) of Theorem 3.1 are satisfied and $f(\theta)$ is continuous at $\theta=0$. Then there exist $C, D>0$ such that

$$
C \sum_{j=0}^{n} x^{2 j}<k_{n}(x, x)<D \sum_{j=0}^{n} x^{2 j}, \quad n \geq N_{1}, x \in I_{n}^{2} \cup I_{n}^{3},
$$

where $N_{1}$ is an integer depending on $C, D$.

Next we give a result of a more general character. Suppose $\left(a_{j}, j \geq 0\right)$ is a sequence of real numbers.

ThEOREM 3.4. If $f(\theta) \leq A<\infty$ we have

$$
E\left(\sum_{j=0}^{m} a_{j} X_{j}\right)^{2} \leq 2 \pi A \sum_{j=0}^{m} a_{j}^{2}, \quad m \geq 0 .
$$

REMARK 3.1. The bound given in Theorem 3.4 was obtained by Yoshihara (1978) when $\left(X_{j}, j \geq 0\right)$ is uniformly mixing with mixing coefficient $\phi(j)$ satisfying the condition $\sum_{j=1}^{\infty} \phi^{1 / 2}(j)<\infty$. 
Proof of TheOREM 3.1. First notice that

$$
k_{n}(x, y)=\int_{-\pi}^{\pi}\left(\sum_{v=0}^{n} x^{v} e^{-i v \theta}\right)\left(\sum_{v=0}^{n} y^{v} e^{i v \theta}\right) f(\theta) d \theta ; x, y \in[0,1) .
$$

This can be seen by expanding the product inside the integral sign. Using (3.1) we deduce that $Q_{n}(x)$ converges in mean square to a function $Q(x)$ for fixed $x \in[0,1)$. Let $k(x, y)=E Q(x) Q(y)$. We have

$$
k(x, y)=\lim _{n \rightarrow \infty} k_{n}(x, y)=\int_{-\pi}^{\pi}\left(1-x e^{-i \theta}\right)^{-1}\left(1-y e^{i \theta}\right)^{-1} f(\theta) d \theta
$$

for $x, y \in[0,1)$.

We construct a sequence of approximations $\left(k^{a}\left(T_{m}, x, y\right), m=0,1, \ldots\right)$ to $k(x, y)$ by replacing $f(\theta)$ in (3.2) by a trigonometric polynomial $T_{m}(\theta)$ of order $m$. Take $m=n$ and $T_{m}(\theta)$ to be the $m$ th partial sum of the Fourier series development of $f(\theta)$. We show that $k^{a}\left(S_{n} f, x, y\right)$ is a good approximation to $k_{n}(x, y)$ for large $n$ and particular $x, y \in I_{n}^{2}$.

We break the proof of Theorem 3.1 into a number of lemmas.

LEMMA 3.1. Let $\left(c_{v}, v=-m, \ldots, m\right)$ be a sequence of real numbers with $c_{v}=c_{-v}, v=1, \ldots, m$, and $T_{m}(\theta)=\sum_{v=-m}^{m} c_{v} e^{i v \theta}$. We have

$$
k^{a}\left(T_{m}, x, y\right)=\frac{G\left(T_{m}, x\right)+G\left(T_{m}, y\right)}{1-x y} ; \quad x, y \in[0,1) .
$$

ProOF. We can write

$$
\begin{aligned}
k^{a}\left(T_{m}, x, y\right)= & \sum_{v=0}^{m} c_{v} \int_{-\pi}^{\pi} e^{i v \theta}\left(1-x e^{-i \theta}\right)^{-1}\left(1-y e^{i \theta}\right)^{-1} d \theta \\
& +\sum_{v=1}^{m} c_{-v} \int_{-\pi}^{\pi} e^{-i v \theta}\left(1-x e^{-i \theta}\right)^{-1}\left(1-y e^{i \theta}\right)^{-1} d \theta .
\end{aligned}
$$

For $v \geq 0$ and $x, y \in[0,1)$ we have by Cauchy's residue theorem that

$$
\int_{-\pi}^{\pi} e^{i v \theta}\left(1-x e^{-i \theta}\right)^{-1}\left(1-y e^{i \theta}\right)^{-1} d \theta=2 \pi x^{v}(1-x y)^{-1}
$$

and

$$
\int_{-\pi}^{\pi} e^{-i v \theta}\left(1-x e^{-i \theta}\right)^{-1}\left(1-y e^{i \theta}\right)^{-1} d \theta=2 \pi y^{v}(1-x y)^{-1}
$$

Lemma 3.1 follows.

The next lemma allows us to determine the behaviour of $k(x, y)$ and $k^{a}\left(T_{m}, x, y\right)$ by the behaviour of $f(\theta)$ and $T_{m}(\theta)$ respectively, in a symmetric 
neighbourhood of $\theta=0$. Let $g(\theta)$ be a real-valued function defined on $\theta \in[-\pi, \pi]$. Suppose that

$$
\int_{-\pi}^{\pi}|g(\theta)| d \theta \leq B<\infty
$$

Define

$$
h(x, y)=\int_{-\pi}^{\pi}\left(1-x e^{-i \theta}\right)^{-1}\left(1-y e^{i \theta}\right)^{-1} g(\theta) d \theta ; \quad x, y \in[0,1) .
$$

LEMMA 3.2. If $\pi \geq b>0$ we have

$$
\left|h(x, y)-\int_{-b}^{b}\left(1-x e^{-i \theta}\right)^{-1}\left(1-y e^{i \theta}\right)^{-1} g(\theta) d \theta\right| \leq C,
$$

where $C$ is a constant depending on $B$ and $b$.

PROOF. Now

$$
\begin{aligned}
& \left|h(x, y)-\int_{-b}^{b}\left(1-x e^{-i \theta}\right)^{-1}\left(1-y e^{i \theta}\right)^{-1} g(\theta) d \theta\right| \\
& \quad \leq 2 \pi B\left(\sup _{|\theta| \in[b, \pi]}\left|1-x e^{-i \theta}\right|^{-1}\right)\left(\sup _{|\theta| \in[b, \pi]}\left|1-y e^{i \theta}\right|^{-1}\right) .
\end{aligned}
$$

The following inequality holds for $x \in[0,1)$.

$$
\begin{aligned}
& \sup _{\pi \geq|\theta| \geq b>0}\left|1-x e^{-i \theta}\right|^{-1} \leq\left(1-\cos ^{2} b\right)^{-1 / 2}, \quad \pi / 2 \geq b>0, \quad \text { and } \\
& \sup _{\pi \geq|\theta| \geq b>0}\left|1-x e^{-i \theta}\right|^{-1} \leq 1, \quad \pi \geq b>\pi / 2 .
\end{aligned}
$$

The desired result follows.

LEMMA 3.3. If $f(\theta) \leq A<\infty$ and $x, y \in[0,1)$ we have

$$
\left|k_{n}(x, y)-k(x, y)\right| \leq(2 \pi A) \frac{\left(x^{n+1}+y^{n+1}+(x y)^{n+1}\right)}{\left(1-x^{2}\right)^{1 / 2}\left(1-y^{2}\right)^{1 / 2}} .
$$

Proof. Sum the series inside the integral sign of (3.1) to give

$$
\begin{aligned}
k_{n}(x, y)= & \int_{-\pi}^{\pi}\left(\frac{1}{\left(1-x e^{-i \theta}\right)}-\frac{\left(x e^{-i \theta}\right)^{n+1}}{\left(1-x e^{-i \theta}\right)}\right) \\
& \times\left(\frac{1}{\left(1-y e^{i \theta}\right)}-\frac{\left(y e^{i \theta}\right)^{n+1}}{\left(1-y e^{i \theta}\right)}\right) f(\theta) d \theta, \quad x, y \in[0,1)
\end{aligned}
$$


Expand the product inside the integral sign of (3.10). Using Cauchy's inequality and $f(\theta) \leq A<\infty$ gives

$$
\begin{aligned}
\left|k_{n}(x, y)-k(x, y)\right| & \leq A\left(x^{n+1}+y^{n+1}+(x y)^{n+1}\right) \\
\times & \left(\int_{-\pi}^{\pi}\left|1-x e^{-i \theta}\right|^{-2} d \theta\right)^{1 / 2}\left(\int_{-\pi}^{\pi}\left|1-y e^{i \theta}\right|^{-2} d \theta\right)^{1 / 2} .
\end{aligned}
$$

Using Lemma 3.1 with $T_{0}(\theta)=1$ gives

$$
k^{a}\left(T_{0}, x, x\right)=\int_{-\pi}^{\pi}\left|1-x e^{-i \theta}\right|^{-2} d \theta=\frac{2 \pi}{\left(1-x^{2}\right)}, \quad x \in[0,1) .
$$

The desired result follows.

We complete the proof of Theorem 3.1. Take $m=n$ and $T_{n}(\theta)=S_{n} f(\theta)$, where $S_{n} f(\theta)$ is the $n$th partial sum of the Fourier series development of $f(\theta)$. Now for $x, y \in[0,1)$ we have

$$
\left|k_{n}(x, y)-k^{a}\left(S_{n} f, x, y\right)\right| \leq J_{1}+J_{2}+J_{3}+J_{4},
$$

where

$$
\begin{aligned}
& J_{1}=\left|k^{a}\left(S_{n} f, x, y\right)-\int_{-b}^{b}\left(1-x e^{-i \theta}\right)^{-1}\left(1-y e^{i \theta}\right)^{-1} S_{n} f(\theta) d \theta\right|, \\
& J_{2}=\left|k(x, y)-\int_{-b}^{b}\left(1-x e^{-i \theta}\right)^{-1}\left(1-y e^{i \theta}\right)^{-1} f(\theta) d \theta\right|, \\
& J_{3}=\left|\int_{-b}^{b}\left(1-x e^{-i \theta}\right)^{-1}\left(1-y e^{i \theta}\right)^{-1}\left(S_{n} f(\theta)-f(\theta)\right) d \theta\right|, \\
& J_{4}=\left|k_{n}(x, y)-k(x, y)\right| .
\end{aligned}
$$

We deduce from condition (ii) of Theorem 3.1 and Zygmund (1958) page 267, Theorem 6.9 that there is a constant $B$ not depending on $n$ such that $\int_{-\pi}^{\pi}\left|S_{n} f(\theta)\right| d \theta \leq B<\infty$, for all $n \geq 0$. Then applying Lemma 3.2 with $g(\theta)=S_{n} f(\theta)$ gives

$$
J_{1} \leq C<\infty,
$$

where $C$ depends on $b$ and $B$. Applying Lemma 3.2 with $g(\theta)=f(\theta)$ gives

$$
J_{2} \leq C<\infty,
$$

where $C$ depends on $b$ and $A$, and where $A$ is an upper bound for $f(\theta)$. Let

$$
a(n)=\sup _{\theta \in[-b, b]}\left|S_{n} f(\theta)-f(\theta)\right| \text {. }
$$

By Cauchy's inequality and (3.12) we have

$$
J_{3} \leq \frac{2 \pi a(n)}{\left(1-x^{2}\right)^{1 / 2}\left(1-y^{2}\right)^{1 / 2}} .
$$


From (3.15), (3.16), (3.17) and Lemma 3.3 we have

$$
\left|k_{n}(x, y)-k^{a}\left(S_{n} f, x, y\right)\right| \leq C\left(1+\frac{a(n)+x^{n+1}+y^{n+1}+(x y)^{n+1}}{\left(1-x^{2}\right)^{1 / 2}\left(1-y^{2}\right)^{1 / 2}}\right)
$$

for $x, y \in[0,1)$ and where $C$ is a constant depending on $b, A$ and $B$. So

$$
\left|k_{n}(x, y)-k^{a}\left(S_{n} f, x, y\right)\right| \leq \frac{w(n)}{\left(1-x^{2}\right)^{1 / 2}\left(1-y^{2}\right)^{1 / 2}}, \quad x, y \in[0,1),
$$

where $w(n) \rightarrow 0, n \rightarrow \infty$ for $x, y \in I_{n}^{1} \cup I_{n}^{2}$.

Now $G\left(S_{n} f, x\right)=\frac{1}{2}+\sum_{v=1}^{n} r_{v} x^{v}$. We show that there is a constant $\alpha>0$ and an integer $N_{0}$ such that

$$
G\left(S_{n} f, x\right) \geq \alpha>0, \quad x \in I_{n}^{2}, n \geq N_{0} .
$$

From Abel's Theorem, Titchmarsh (1939), page 9 and condition (i) of Theorem 3.1 we see that $G\left(S_{n} f, x\right)$ is uniformly convergent for $x \in[0,1]$ and

$$
\lim _{x \rightarrow 1^{-}} G\left(S_{\infty} f, x\right)=\frac{1}{2}+\sum_{v=1}^{\infty} r_{v}=\pi f(0) .
$$

Condition (3.20) follows from the uniform convergence of $G\left(S_{n} f, x\right),(3.21)$ and condition (iii) of Theorem 3.1. The conclusions of Theorem 3.1 follow.

ProOF OF COROLlary 3.1. Now by simple manipulation we have

$$
\begin{aligned}
\frac{r_{n}(x, y)}{r^{\prime}(x, y)}= & \left\{\frac{k_{n}(x, y) / k^{a}\left(S_{n} f, x, y\right)}{\left(k_{n}(x, x) / k^{a}\left(S_{n} f, x, x\right)\right)^{1 / 2}\left(k_{n}(y, y) / k^{a}\left(S_{n} f, y, y\right)\right)^{1 / 2}}\right\} \\
& \times\left\{\frac{G\left(S_{n} f, x\right)+G\left(S_{n} f, y\right)}{\left(4 G\left(S_{n} f, x\right) G\left(S_{n} f, y\right)\right)^{1 / 2}}\right\}
\end{aligned}
$$

Proof of Theorem 3.2. Conditions (ii) and (iii) of Theorem 3.1 follow from the uniform convergence of $S_{n} f(\theta), \theta \in[-\pi, \pi]$, and $\pi S_{n} f(0)=G\left(S_{n} f, 0\right)$. Following the proof of Theorem 3.1 we see that $J_{1}=J_{2}=0$. So (3.19) holds for $x \in I_{n}^{1} \cup I_{n}^{2}$. The desired result follows as (3.20) holds for $x \in I_{n}^{1} \cup I_{n}^{2}$ by assumption.

PROOF OF THEOREM 3.3. For some $\mu$ we construct an interval $[-b, b]$, $\pi \geq b>0$, where $f(\theta) \geq \mu>0, \theta \in[-b, b]$. Such an interval exists as $f(\theta)$ is continuous at $\theta=0$ and $f(0)>0$. We consider the case when $x \in$ $\left[1-(\log n)^{-1 / 2}, 1\right)$ and $x=1$ separately. Use $(3.1)$ to represent $k_{n}(x, x)$. As (3.9) holds for

$$
\sup _{\pi \geq|\theta| \geq b>0}\left|\sum_{v=0}^{n} x^{v} e^{-i v \theta}\right|, \quad x \in[0,1)
$$


we can apply Lemma 3.2 to $k_{n}(x, x)$ with $\left|1-x e^{-i v \theta}\right|^{2}$ replaced by $\left|\sum_{v=0}^{n} x^{v} e^{-i v \theta}\right|^{2}$ to give

$$
\left.\left|k_{n}(x, x)-\int_{-b}^{b}\right| \sum_{v=0}^{n} x^{v} e^{-i v \theta}\right|^{2} f(\theta) d \theta \mid \leq C, \quad x \in[0,1) .
$$

Substitute $f(\theta)=1 / 2 \pi$ in (3.23) to give

$$
\left.\left|\int_{-\pi}^{\pi}\right| \sum_{v=0}^{n} x^{v} e^{-i v \theta}\right|^{2} d \theta-\int_{-b}^{b}\left|\sum_{v=0}^{n} x^{v} e^{-i v \theta}\right|^{2} d \theta \mid \leq C,
$$

where $C$ depends only on $b$ and $A$. These inequalities follow in the same way as Lemma 3.2.

By simple calculation we have

$$
\int_{-\pi}^{\pi}\left|\sum_{v=0}^{n} x^{v} e^{-i v \theta}\right|^{2} d \theta=2 \pi \sum_{v=0}^{n} x^{2 v}, \quad x \in[0,1] .
$$

From (3.25) and (3.24) we have

$$
\int_{-b}^{b}\left|\sum_{v=0}^{n} x^{v} e^{-i v \theta}\right|^{2} d \theta \sim 2 \pi \sum_{v=0}^{n} x^{2 v}, \quad n \rightarrow \infty
$$

and $x \in\left[1-(\log n)^{-1 / 2}, 1\right)$. From our construction of $[-b, b]$ we have

$$
\int_{-b}^{b}\left|\sum_{v=0}^{n} x^{v} e^{-i v \theta}\right|^{2} f(\theta) d \theta \geq \mu \int_{-b}^{b}\left|\sum_{v=0}^{n} x^{v} e^{-i v \theta}\right|^{2} d \theta .
$$

So the desired result follows from $(3.27),(3.26)$ and $(3.23)$ for $x \in\left[1-(\log n)^{-1 / 2}, 1\right)$.

When $x=1$ we have

$$
k_{n}(1,1)=n+1+2 \sum_{j=1}^{n}(n-j+1) r_{j}=2 \pi(n+1) \sigma_{n} f(0) .
$$

where $\sigma_{n} f(\theta)$ is the $n$th Cesàro sum associated with $f(\theta)$. As $f(\theta)$ is continuous at $\theta=0$ we have $\sigma_{n} f(0) \rightarrow f(0), n \rightarrow \infty$. The desired result follows.

ProOF OF ThEOREM 3.4. Now

$$
E\left(\sum_{j=0}^{m} a_{j} X_{j}\right)^{2}=\int_{-\pi}^{\pi}\left(\sum_{v=0}^{m} a_{v} e^{-i v \theta}\right)\left(\sum_{v=0}^{m} a_{v} e^{i v \theta}\right) f(\theta) d \theta .
$$

Note that $f(\theta) \leq A<\infty$. Then by Cauchy's inequality we have

$$
E\left(\sum_{j=0}^{m} a_{j} X_{j}\right)^{2} \leq A \int_{-\pi}^{\pi}\left|\sum_{v=0}^{m} a_{v} e^{-i v \theta}\right|^{2} d \theta .
$$


By simple calculation we have

$$
\int_{-\pi}^{\pi}\left|\sum_{v=0}^{m} a_{v} e^{-i v \theta}\right|^{2} d \theta=2 \pi \sum_{j=0}^{m} a_{j}^{2} .
$$

The desired result follows from (3.30) and (3.31).

\section{Approximation}

For $x \in[a, b]$ we approximate $Q_{n}(x)$ by a process which linearly interpolates between $Q_{n}(a)$ and $Q_{n}(b)$. It is convenient to count the sign changes of this process in $[a, b]$ by

$$
\hat{N}_{n}(a, b)=\left(\frac{1}{2}\right)-\left(\frac{1}{2}\right) \operatorname{sgn}\left(Q_{n}(a) Q_{n}(b)\right),
$$

where $\operatorname{sgn} x=x /|x|, x \neq 0$ and $\operatorname{sgn} 0=0$. The results of this section depend on an upper bound for the number of zeros of $Q_{n}(x)$ in an interval $[a, b]$. Define the event

$$
U_{k}=\left(Q_{n}(x) \text { has } k \text { or more zeros in }[a, b]\right), \quad k \geq 0 .
$$

For any interval $[a, b] \subseteq[0,1]$ let

$$
\begin{aligned}
& \gamma=(b-a)(1-b)^{-1}, \quad b \in\left[0,1-(n+1)^{-1}\right], \quad \text { and } \\
& \gamma=(n+1)(b-a), \quad b \in\left[1-(n+1)^{-1}, 1\right] .
\end{aligned}
$$

LEMMA 4.1. Provided $\gamma<2^{-30}$ and

(i) $f(\theta)$ is continuous at $\theta=0$,

(ii) $f(0)>0$,

(iii) $f(\theta) \leq A<\infty, \theta \in[-\pi, \pi]$,

then there is an integer $N_{1}$ and an absolute constant $C$ such that

$$
P\left(U_{k}\right)<C \gamma^{3 k / 5}, \quad n \geq N_{1}, k \geq 0 .
$$

COROLLARY 4.1. Under the conditions of Lemma 4.1 we have

$$
\left|E \hat{N}_{n}(a, b)-E N_{n}([a, b])\right|<C \gamma^{6 / 5}, \quad n \geq N_{1},
$$

where $C$ is an absolute constant.

ProOF OF LEMMA 4.1. Let $t>0$. Then

$$
P\left(U_{k}\right) \leq P\left(\frac{\left|Q_{n}(b)\right|}{\left(k_{n}(b, b)\right)^{1 / 2}}<t\right)+P\left(U_{k} \cap\left(\frac{\left|Q_{n}(b)\right|}{\left(k_{n}(b, b)\right)^{1 / 2}} \geq t\right)\right) .
$$

We estimate the second term in (4.3). Let

$$
Q_{n}^{(k)}(x)=\sum_{j=k}^{n} a_{j k}(x) X_{j} ; \quad a_{j k}(x)=\frac{d^{k}}{d x^{k}} x^{j}, \quad k \leq j .
$$


In the same way as (2.13) of Ibragimov and Masolva (1971a) page 233, we have

$$
P\left(U_{k} \cap \frac{\left|Q_{n}(b)\right|}{\left(k_{n}(b, b)\right)^{1 / 2}} \geq t\right) \leq \frac{1}{t^{2}}\left[\frac{(b-a)^{k}}{k !}\right]^{2} \frac{\sup _{x \in[a, b]} E\left(Q_{n}^{(k)}(x)\right)^{2}}{\left(k_{n}(b, b)\right)}
$$

Applying Theorem 3.4 to $E\left(Q_{n}^{(k)}(x)\right)^{2}$ and using the upper bound of Stevens (1969), Lemma 9, gives

$$
E\left(Q_{n}^{(k)}(x)\right)^{2} \leq A \sum_{j=k}^{n} a_{j k}^{2}(x)<A(2 k) !\left(\sum_{j=0}^{n} x^{2 j}\right)^{2 k+1}, \quad x \in[0,1] .
$$

From Theorem 3.3 there is an integer $N_{1}$ and a constant $C$ such that

$$
k_{n}(b, b)>C \sum_{j=0}^{n} b^{2 j}, \quad n \geq N_{1}, b \in\left[1-(\log n)^{-1 / 2}, 1\right] .
$$

Take $n \geq N_{1}$. From (4.5) and (4.6) we have

$$
P\left(U_{k} \cap \frac{\left|Q_{n}(b)\right|}{\left(k_{n}(b, b)\right)^{1 / 2}} \geq t\right)<C \frac{1}{t^{2}}\left[\frac{(b-a)^{k}}{k !}\right]^{2}(2 k) !\left(\sum_{j=0}^{n} b^{2 j}\right)^{2 k} .
$$

Take $t=\gamma^{2 k / 3}$. Substitute in (4.7) for $(b-a)$ given by (4.2) where

$$
b \in\left[1-(\log n)^{-1 / 2}, 1-(n+1)^{-1}\right] \text { and } b \in\left[1-(n+1)^{-1}, 1\right] .
$$

By Stirling's Theorem (Titchmarsh (1939), page 58) there is a constant $C$ such that $(2 k) ! /(k !)^{2}<C 2^{2 k}$. So

$$
P\left(U_{k} \cap \frac{\left|Q_{n}(b)\right|}{\left(k_{n}(b, b)\right)^{1 / 2}} \geq \gamma^{2 k / 3}\right)<C 2^{2 k} \gamma^{2 k / 3}<C\left(2^{2} \gamma^{1 / 15}\right)^{k} \gamma^{3 k / 5},
$$

for $n \geq N_{1}$ and $b \in I_{n}^{2} \cup I_{n}^{3}$. The desired result follows as $\gamma<2^{-30}$.

We estimate the first term in (4.3) by noting that $Q_{n}(x)$ is distributed as a normal random variable with zero mean and variance $k_{n}(x, x)$. So

$$
P\left(\frac{\left|Q_{n}(b)\right|}{\left(k_{n}(b, b)\right)^{1 / 2}}<\gamma^{2 k / 3}\right)<(1 / 2 \pi)^{1 / 2} \gamma^{2 k / 3} .
$$

The desired result follows.

ProOF OF COROLlaRY 4.1. From the definition of $\hat{N}_{n}(a, b)$ we have

$$
\left|E \hat{N}_{n}(a, b)-E N_{n}([a, b])\right| \leq 2 \sum_{k=2}^{n} P\left(U_{k}\right) .
$$

The desired result follows immediately. 


\section{An estimate for $E N_{n}\left(I_{n}^{2}\right)$}

In this section we give sufficient conditions for $E N_{n}\left(I_{n}^{2}\right) \sim(1 / 2 \pi) \log n$ as $n$ tends to infinity. These conditions are satisfied by the Gaussian processes specified in Theorems 2.1, 2.2 and 2.3.

LEMMA 5.1. Suppose that $0<b \leq \pi$. If

(i) $f(\theta)$ can be uniformly approximated in $[-b, b]$ by the partial sums of its Fourier series development, $S_{n} f(\theta)$,

(ii) $f(0)>0$,

(iii) $f(\theta) \leq A<\infty, \theta \in[-\pi, \pi]$,

then $E N_{n}\left(I_{n}^{2}\right) \sim(1 / 2 \pi) \log n$ as $n \rightarrow \infty$.

ProOF. For fixed $\delta \in\left(0,2^{-30}\right)$ define the set $P=\left(x_{j}, j \geq 0\right)$ where

$$
x_{j}=1-\frac{1}{(1+\delta)^{j}} .
$$

Let $p_{0}^{n}, p_{1}^{n}$ be positive integers satisfying the inequalities

$$
\begin{aligned}
\frac{1}{(1+\delta)^{p_{0}^{n}}} & \leq \frac{1}{(\log n)^{1 / 2}}<\frac{1}{(1+\delta)^{p_{0}^{n}-1}}, \\
\frac{1}{(1+\delta)^{p_{1}^{n}+1}} & <\frac{\log \log n}{n} \leq \frac{1}{(1+\delta)^{p_{1}^{n}}}, \quad n \geq 3 .
\end{aligned}
$$

We estimate the asymptotic growth of $\sum_{j=p_{0}^{n}}^{p_{1}^{n}-1} E \hat{N}_{n}\left(x_{j}, x_{j+1}\right)$ and show that it is a good approximation to the growth of $E N_{n}\left(I_{n}^{2}\right)$.

From Dunnage (1966) Lemma 4 we have

$$
E \hat{N}_{n}\left(x_{j}, x_{j+1}\right)=(1 / \pi) \sin ^{-1}\left(\left(1-r_{n}\left(x_{j}, x_{j+1}\right)^{2}\right)^{1 / 2}\right), \quad j \geq 0 .
$$

By simple calculation we have

$$
\sup _{j=p_{0}^{n}, \ldots, p_{1}^{n}-1}\left|r^{\prime}\left(x_{j}, x_{j+1}\right)-2 \frac{(1+\delta)^{1 / 2}}{2+\delta}\right| \rightarrow 0, \quad n \rightarrow \infty .
$$

So by Corollary 3.1 we have

$$
\sup _{j=p_{0}^{n}, \ldots, p_{1}^{n}-1}\left|\sin ^{-1}\left(\left(1-r_{n}\left(x_{j}, x_{j+1}\right)^{2}\right)^{1 / 2}\right)-\sin ^{-1}\left(\frac{\delta}{2+\delta}\right)\right| \rightarrow 0, \quad n \rightarrow \infty .
$$

Now there is an absolute constant $C$ such that

$$
\left|\sin ^{-1}\left(\frac{\delta}{2+\delta}\right)-\delta / 2\right| \leq C \delta^{2} / 2, \quad \infty>C>0, \delta \in\left[0,2^{-15}\right) .
$$


We choose an integer $N_{2}$ so that the left-hand side of (5.6) is at most $C \delta^{2} / 2$ for $n \geq N_{2}$. From (5.7), (5.6) and (5.4) we have

$$
\sup _{j=p_{0}^{n}, \ldots, p_{1}^{n}-1}\left|E \hat{N}_{n}\left(x_{j}, x_{j+1}\right)-\delta / 2 \pi\right| \leq C \delta^{2}, \quad n \geq N_{2} .
$$

From (5.2) and (5.3) there is an integer $N_{3}$ such that

$$
\left|\frac{p_{1}^{n}-p_{0}^{n}}{\log n / \delta}-1\right| \leq \varepsilon, \quad n \geq N_{3}, \text { for any } \varepsilon>0
$$

So

$$
\left|\sum_{j=p_{0}^{n}}^{p_{1}^{n}-1} \frac{E \hat{N}_{n}\left(x_{j}, x_{j+1}\right)}{\log n / 2 \pi}-1\right| \leq \varepsilon+2 \pi(1+\varepsilon) \delta C, \quad n \geq \max \left(N_{2}, N_{3}\right) .
$$

By our choice of $\delta$ and $\varepsilon$ we can make the right-hand side of (5.10) arbitrarily small. So

$$
\sum_{j=p_{0}^{n}}^{p_{1}^{n}-1} E \hat{N}_{n}\left(x_{j}, x_{j+1}\right) \sim(1 / 2 \pi) \log n, \quad n \rightarrow \infty .
$$

From condition (i) of Lemma 5.1 we see that $f(\theta)$ is continuous in $(-b, b)$. Then applying Corollary 4.1 with $\gamma=\delta$ for $\left[x_{j}, x_{j+1}\right], j=p_{0}^{n}, \ldots, p_{1}^{n}-1$, gives

$$
\left|\sum_{j=p_{0}^{n}}^{p_{1}^{n}-1} E N_{n}\left(\left[x_{j}, x_{j+1}\right]\right)-\sum_{j=p_{0}^{n}}^{p_{1}^{n}-1} E \hat{N}_{n}\left(x_{j}, x_{j+1}\right)\right| \leq C \delta^{6 / 5}\left(p_{1}^{n}-p_{0}^{n}\right),
$$

$$
n \geq N_{1} \text {. }
$$

In the following intervals we have $\gamma \leq \delta$. Then from Lemma 4.1 we have

$$
E N_{n}\left(\left[x_{p_{1}^{n}}, 1-n^{-1} \log \log n\right]\right) \leq C \delta^{6 / 5}
$$

and

$$
E N_{n}\left(\left[1-(\log n)^{-1 / 2}, x_{p_{0}^{n}}\right]\right) \leq C \delta^{6 / 5} \text { for } n \geq N_{1} .
$$

From (5.12), (5.13) and $P\left(Q_{n}(x)=0\right)=0$ we have

$$
\left|E N_{n}\left(I_{n}^{2}\right)-\sum_{j=p_{0}^{n}}^{p_{1}^{n}-1} E \hat{N}_{n}\left(x_{j}, x_{j+1}\right)\right| \leq C \delta^{6 / 5}\left(p_{1}^{n}-p_{0}^{n}+2\right), \quad n \geq N_{1},
$$

where $C$ is a constant. From (5.9) and (5.14) we have

$$
\begin{array}{r}
\left|\frac{E N_{n}\left(I_{n}^{2}\right)}{\log n / 2 \pi}-\sum_{j=p_{0}^{n}}^{p_{1}^{n}-1} \frac{E \hat{N}_{n}\left(x_{j}, x_{j+1}\right)}{\log n / 2 \pi}\right| \leq \frac{4 \pi C \delta^{6 / 5}}{\log n}+2 \pi C \delta^{1 / 5}(1+\varepsilon), \\
n \geq \max \left(N_{1}, N_{3}\right) .
\end{array}
$$


The desired result follows from (5.10) and (5.15) by taking $\delta$ and $\varepsilon$ arbitrarily small.

\section{An estimate for $E N_{n}\left(I_{n}^{1}\right)$ and $E N_{n}\left(I_{n}^{3}\right)$}

In this section we show that $E N_{n}\left(I_{n}^{1}\right)$ and $E N_{n}\left(I_{n}^{3}\right)$ are $o(\log n)$ as $n$ tends to infinity under the conditions of Theorems $2.1,2.2$ and 2.3.

LEMMA 6.1. Under the conditions of Theorem 2.1 there is a finite constant $C$ such that

$$
E N_{n}\left(I_{n}^{1}\right) \leq\left(C^{1 / 2} / 2 \pi\right) \log \log n, \quad n \geq 2
$$

PROOF. The proof of this result is based on the Kac-Rice formula (Shenker (1981), page 511, or Kac (1948)) which states that

$$
E N_{n}([a, b])=(1 / \pi) \int_{a}^{b} C_{n}^{1 / 2} B_{n}^{-1 / 2}\left(1-R_{n}^{2}\right)^{2} d x
$$

where

$$
\begin{aligned}
& B_{n}=E\left(Q_{n}(x)\right)^{2}, \quad C_{n}=E\left(\frac{d}{d x} Q_{n}(x)\right)^{2} \text { and } \\
& R_{n}=\operatorname{Cor}\left(Q_{n}(x), \frac{d}{d x} Q_{n}(x)\right) .
\end{aligned}
$$

We find an upper bound for $C_{n} / B_{n}$ with $x \in I_{n}^{1}$ and apply (6.1) to obtain the desired result.

We represent $B_{n}=k_{n}(x, x)$ by (3.1). Noting that $f(\theta) \geq m>0, \theta \in[-\pi, \pi]$, and applying (3.25) gives

$$
B_{n} \geq 2 \pi m \sum_{v=0}^{n} x^{2 v}, \quad x \in[0,1] .
$$

From Theorem 3.4 we have $C_{n} \leq 2 \pi A \sum_{v=1}^{n} v^{2} x^{2(v-1)}$. Summing $\sum_{v=0}^{n} x^{2 v}$ and noting that

$$
\sum_{v=1}^{n} v^{2} x^{2(v-1)} \leq 2\left(1-x^{2}\right)^{-3}, \quad x \in[0,1)
$$

gives

$$
C_{n} / B_{n} \leq(2 A / m)\left(1-x^{2(n+1)}\right)^{-1}\left(1-x^{2}\right)^{-2} .
$$

As $x^{2(n+1)}<1, x \in I_{n}^{1}, n \geq 2$ and $\sup _{x \in I_{n}^{1}} x^{2(n+1)} \rightarrow 0, x \in I_{n}^{1}, n \rightarrow \infty$, we deduce that $\left(1-x^{2(n+1)}\right)^{-1}$ is bounded above by a constant. So

$$
C_{n} / B_{n} \leq(1-x)^{-2}, \quad n \geq 2 \text { and } x \in I_{n}^{1} .
$$


Substituting for $C_{n} / B_{n}$ in (6.1) and noting that $\left(1-R_{n}^{2}\right) \leq 1$ gives the desired result.

LEMMA 6.2. Under the conditions of Theorem 2.2 there is a constant $C$ and an integer $N_{5}$ such that

$$
E N_{n}\left(I_{n}^{1}\right) \leq\left(C^{1 / 2} / 2 \pi\right) \log \log n, \quad n \geq N_{5} .
$$

ProOF. Again we find an upper bound for $C_{n} / B_{n}, x \in I_{n}^{1}$ and apply (6.1). Note that $G\left(S_{n} f, x\right)=\frac{1}{2}+\sum_{j=1}^{n} r_{j} x^{j} \geq \alpha>0$ for $n \geq N_{0}$. So the conditions of Theorem 3.2 are satisfied. From Theorem 3.2 and any $C \in(0,1)$ we have an integer $N_{4}$ such that

$$
B_{n}=k_{n}(x, x)>C k^{a}\left(S_{n} f, x, x\right), \quad n \geq N_{4}, x \in I_{n}^{1} .
$$

From Lemma 3.1 we have

$$
k^{a}\left(S_{n} f, x, x\right)=2 \frac{G\left(S_{n} f, x\right)}{1-x^{2}} .
$$

But $G\left(S_{n} f, x\right) \geq \alpha>0$ for $n \geq N_{0}$. So

$$
B_{n}>\frac{2 \alpha C}{1-x^{2}}, \quad n \geq N_{5}=\max \left(N_{0}, N_{4}\right) .
$$

In the same way as in Lemma 6.1 we have

$$
B_{n} / C_{n} \leq(1-x)^{-2}, \quad n \geq N_{5}=\max \left(N_{0}, N_{4}\right) .
$$

The desired result follows.

We need the following inequality.

LEMMA 6.3 (Ibragimov (1962), Lemma 1.9, p. 361). Let $\left(Z_{j}, j \geq 0\right)$ be a stationary sequence of uniformly mixing random variables with zero mean and

(i) $E\left|Z_{j}\right|^{2+\delta}<\infty, \delta>0$.

(ii) $E\left(\sum_{j=0}^{n} Z_{j}\right)^{2} \rightarrow \infty, n \rightarrow \infty$.

Then there exists a constant $C$ such that

$$
E\left|\sum_{j=0}^{n} Z_{j}\right|^{2+\delta} \leq C\left|E\left(\sum_{j=0}^{n} Z_{j}\right)^{2}\right|^{1+\delta / 2} .
$$

LEMMA 6.4. Under the conditions of Theorem 2.3 we have

$$
E N_{n}\left(I_{n}^{1}\right) \leq C(\log n)^{1 / 2} \log \log n,
$$

where $C$ is a constant.

Proof. Choose $c$ such that

$$
P\left(\left|X_{0}\right| \geq c\right)=q<1 .
$$


Define the events $B_{0}=\left(\left|X_{0}\right|<c\right)$ and

$$
\begin{aligned}
B_{k} & =\left(\left|X_{0}\right|<c, \ldots,\left|X_{k-1}\right|<c,\left|X_{k}\right| \geq c\right), \quad k=1, \ldots, n . \\
B & =\left(\left|X_{0}\right|<c, \ldots,\left|X_{n}\right|<c\right) .
\end{aligned}
$$

Let $0<r<R$. Following the argument of Ibragimov and Maslova (1971a) leading to the relationship (2.6) we obtain

$$
E N_{n}([-r, r]) \leq \sum_{k=1}^{n} k P\left(B_{k}\right)+n P(B)+(\log R / r)^{-1} \sum_{k=0}^{n} \int_{B_{k}} H_{s} d P
$$

where

$$
H_{s}=\log \left(\sup _{\theta \in[-\pi, \pi]}(k ! c)^{-1}\left|Q_{n}^{(k)}\left(R e^{i \theta}\right)\right|\right) .
$$

Here $Q_{n}^{(k)}(x)$ is the $k$ th derivative of $Q_{n}(x)$ with respect to $x$.

We estimate $P\left(B_{k}\right)$ and $P(B)$. Define a sequence of random variables $\left(Z_{k}\right.$, $k=0, \ldots, n)$ by

$$
Z_{k}=1-q, \quad\left|X_{k}\right| \geq c \quad \text { and } \quad Z_{k}=-q, \quad\left|X_{k}\right|<c .
$$

Now for $k=1, \ldots, n$ we have

$$
\begin{aligned}
B_{k} & =\left(\left(\sum_{j=0}^{k-1} Z_{j}=-k q\right) \cap\left(Z_{k}=1-q\right)\right) \text { and } \\
B_{0} & =\left(Z_{0}=1-q\right) .
\end{aligned}
$$

We show that the conditions of Lemma 6.3 are satisfied by $\left(Z_{k}, k \geq 0\right)$ with $\delta=4$. Clearly $\left(Z_{k}, k \geq 0\right)$ is a stationary sequence of uniformly mixing random variables with mixing coefficient $\phi(j)$. Using Ibragimov (1962), page 364, and $\sum_{j=1}^{\infty} \phi^{1 / 2}(j)<\infty$ gives

$$
E\left(\sum_{j=0}^{n-1} Z_{j}\right)^{2} \sim n, \quad n \rightarrow \infty
$$

So condition (ii) is satisfied. Condition (i) is immediate.

Using (6.15), Markov's inequality and Lemma 6.3 with $\delta=4$ gives

$$
P\left(B_{k}\right) \leq P\left(\left|\sum_{j=0}^{k-1} Z_{j}\right| \geq k q / 2\right)<C / k^{3}, \quad C<\infty, k \geq 1,
$$

and

$$
\sum_{k=1}^{\infty} k P\left(B_{k}\right)<\infty
$$


In the same way

$$
P(B) \leq P\left(\left|\sum_{j=0}^{n} Z_{j}\right| \geq q(n+1) / 2\right)<C / n^{3}, \quad C<\infty,
$$

and

$$
n P(B) \rightarrow 0, \quad n \rightarrow \infty
$$

We estimate the final term in (6.12) using the method of Ibragimov and Maslova (1971a), Lemma 1. Let $T>0$. Then

$$
\int_{B_{k}} H_{s} d P \leq P\left(B_{k}\right) \log w_{k}+P\left(B_{k}\right) \log T+C\left(1+i_{0}\right) \exp \left(-i_{0}\right)
$$

where $C$ is a constant, $i_{0}=\log T$ and

$$
W_{k}=E\left(\sum_{j=k}^{n} \frac{j(j-1) \cdots(j-k+1) R^{j-k}}{k ! c}\left|X_{j}\right|\right) .
$$

Taking $T$ to be the following function of $k$,

$$
T=1, \quad k=0 ; \quad T=k^{1+\varepsilon}, \quad k \geq 1, \quad \varepsilon>0,
$$

in (6.1) and noting that $W_{k}<C(1-R)^{-k-1} c^{-1}, 0 \leq R<1$ we have

$$
\begin{aligned}
\sum_{k=0}^{n} \int_{B_{k}} H_{s} d P \leq & \left(\log \frac{C}{1-R}\right)\left(\sum_{k=0}^{n}(k+1) P\left(B_{k}\right)\right) \\
& +(1+\varepsilon) \sum_{k=1}^{n}(\log k) P\left(B_{k}\right)+D \sum_{k=1}^{n} \frac{1+(1+\varepsilon) \log k}{k^{1+\varepsilon}}-\log c
\end{aligned}
$$

where $C$ and $D$ are constants. Substitute $r=1-(\log n)^{-1 / 2}$ and $R=1-$ $\frac{1}{2}(\log n)^{-1 / 2}$ in (6.24). Using (6.17) and noting (6.18) and (6.20) gives the desired result.

Next we estimate the growth of $E N_{n}\left(I_{n}^{3}\right)$.

LEMMA 6.5. If

(i) $f(\theta)$ is continuous at $\theta=0$,

(ii) $f(0)>0$,

(iii) $f(\theta) \leq A<\infty, \theta \in[-\pi, \pi]$,

then there is a constant $C$ and an integer $N_{6}$ such that

$$
E N_{n}\left(I_{n}^{3}\right)<C(\log \log n)^{7 / 5}, \quad n \geq N_{6} .
$$


ProOF. Define $p=((n+1) \log \log n)^{-1}$. Partition $I_{n}^{3}$ into intervals $\left(J_{s}\right.$, $s=1, \ldots, v)$ where

$$
\begin{aligned}
& J_{s}=\left[1-n^{-1} \log \log n+(s-1) p, 1-n^{-1} \log \log n+s p\right], \\
& \quad \text { for } s=1, \ldots, v-1, \\
& J_{v}=\left[1-n^{-1} \log \log n+(v-1) p, 1\right], \quad n \geq 3 .
\end{aligned}
$$

If $[x]$ is the integer part of $x$ we have

$$
v=\left[((n+1) / n)(\log \log n)^{2}\right]+1 .
$$

For $U_{k}$ defined in Section 4 we have

$$
E N_{n}\left(J_{s}\right) \leq \sum_{k=1}^{n} P\left(U_{k}\right), \quad s=1, \ldots, v .
$$

Applying Lemma 4.1 to $J_{s}$ and noting that $\gamma<(\log \log n)^{-1}$ gives

$$
E N_{n}\left(J_{s}\right) \leq C \sum_{k=1}^{n} \gamma^{3 k / 5}=C \gamma^{3 / 5}\left(1-\gamma^{3 / 5}\right)^{-1}, \quad n \geq N_{6},
$$

where $N_{6}>\max \left(N_{1}, \exp \left(\exp \left(2^{30}\right)\right)\right)$. So $\gamma<2^{-30}$. Using (6.26), (6.28) and noting that $P\left(Q_{n}(x)=0\right)=0$ gives

$$
E N_{n}\left(I_{n}^{3}\right)=\sum_{s=1}^{v} E N_{n}\left(J_{s}\right)<C \frac{\gamma^{3 / 5}}{1-\gamma^{3 / 5}}(1+1 / n)(\log \log n)^{2}
$$

for $n \geq N_{6}$. The desired result follows.

ProOF OF THEOREMS 2.1, 2.2 AND 2.3. We show that

$$
E N_{n}\left(I_{n}^{1}\right)=o(\log n), \quad E N_{n}\left(I_{n}^{3}\right)=o(\log n)
$$

and

$$
E N_{n}\left(I_{n}^{2}\right) \sim(1 / 2 \pi) \log n, \quad n \rightarrow \infty .
$$

We have already shown that $E N_{n}\left(I_{n}^{1}\right)=o(\log n), n \rightarrow \infty$, under the conditions of Theorems 2.1, 2.2 and 2.3. The remaining relationships hold when the conditions of Lemmas 6.5 and 5.1 are satisfied. Notice that the conditions of Lemma 6.5 are contained in those of Lemma 5.1. So Theorems 2.1, 2.2 and 2.3 follow when the conditions of Lemma 5.1 are satisfied. This is immediate for Theorem 2.1. For Theorem 2.2 we note that the uniform convergence of $S_{n} f(\theta)$ to $f(\theta), \theta \in[-\pi, \pi]$, implies condition (i) and (iii) of Lemma 5.1. Now

$$
S_{n} f(0)=(1 / \pi)\left(\frac{1}{2}+\sum_{j=1}^{n} r_{j}\right) \geq \alpha / \pi>0,
$$

so condition (ii) of Lemma 5.1 follows. 
Consider the conditions of Theorem 2.3. From Ibragimov, (1962) page 364, and (1.1) it follows that

$$
\left|r_{j}\right| \leq 2 \phi^{1 / 2}(j), \quad j \geq 1 .
$$

So under the conditions of Theorem 2.3 we have

$$
\sum_{j=1}^{\infty}\left|r_{j}\right|<\infty
$$

We deduce that $S_{n} f(\theta)$ converges uniformly to $f(\theta), \theta \in[-\pi, \pi]$. So conditions (i) and (iii) of Lemma 5.1 are satisfied. Condition (ii) holds by assumption.

Proof of COROLlary 2.1. As $\left(X_{j}, j \geq 0\right)$ is stationary we have

$$
E N_{n}([1, \infty))=E N_{n}([0,1])
$$

and

$$
E N_{n}((-\infty, 1])=E N_{n}([-1,0]) \text {. }
$$

We have already shown that $E N_{n}([0,1]) \sim(2 \pi)^{-1} \log n, n \rightarrow \infty$. So Corollary 2.1 follows if

$$
E N_{n}([-1,0]) \sim(2 \pi)^{-1} \log n, \quad n \rightarrow \infty .
$$

Now $Q_{n}(x)$ for $x \in[-1,0]$ can be written as

$$
Q_{n}(-x)=\sum_{j=0}^{n}(-1)^{j} X_{j} x^{j}, \quad x \in[0,1] .
$$

The spectral density associated with $\left((-1)^{j} X_{j}, j \geq 0\right)$ is $f(\theta+\pi)$, so the desired result follows.

\section{Comparison with earlier work}

Sufficient conditions for (1.3) are given in Theorems 1 and 3 of Shenker (1981) when $\left(X_{j}\right)$ is a stationary Gaussian process satisfying (1.1).

The principal result is Theorem 1 which states that $\sum_{j=0}^{\infty}\left|r_{j}\right|<\frac{1}{2}$ is sufficient for (1.3) to hold. This result is contained in Corollary 2.1.

Theorem 3 of Shenker (1981) states that (1.3) holds when $\left(\left|r_{j}\right|, j \geq 0\right)$ is strictly convex (that is, $\left.\left|r_{j+2}\right|-2\left|r_{j+1}\right|+\left|r_{j}\right|>0\right) ; r_{2 j}>0$ and $\sum_{j=1}^{n} j\left|r_{j}\right|=o(n)$ as $n$ tends to infinity.

Define the sequence $\left(r_{j}, j \geq 0\right)$ by

$$
r_{j}=(j+1)^{-1}(\log (j+1)+1)^{-1}, \quad j \geq 0 .
$$

This sequence is an autocorrelation function of a stationary process. This follows from the strict convexity of $\left(r_{j}, j \geq 0\right)$ and Zygmund (1958), Theorem 1.5, page 
183. Since $\left(r_{j}, j \geq 0\right)$ satisfies the conditions of Theorem 3 yet $S_{n} f(0) \rightarrow \infty$, $n \rightarrow \infty$ this process is not contained in any of our results.

Sufficient conditions for $E N_{n}([0,1]) \sim(1 / 2 \pi) \log n$ as $n$ tends to infinity are given in Theorem 2 of Shenker (1981) when $r_{j} \geq 0, j \geq 1$. Our construction (6.37) satisfies the conditions of this result yet is not contained in any of our results.

Suppose that $\left(X_{j}, j=0, \ldots, n\right)$ is distributed as a multivariate normal random vector satisfying (1.1). Let $r_{i j}=E X_{i} X_{j}$. Take $r \in(0,1)$. The case where $r_{i j}=r$ and $r_{i j}=r^{|i-j|}$, for all $i, j$ (with $i \neq j$ ) are considered in Sambandham $(1979,1977)$ respectively. In the first case $E N_{n}((-\infty, \infty)) \sim(1 / 2) \log n$ as $n$ tends to infinity and in the second (1.3) holds when $0<r<1 / 2$.

By calculating $f(\theta)$ we see that the second case is contained in Corollary 2.1. By considering $\sum_{j=1}^{n} r_{j}$ we see that no Gaussian process with $r_{j}=r, j \geq 1$, $r \in(0,1)$ can be included in any of our results.

\section{References}

A. T. Bharucha-Reid and M. Sambandham (1986), Random polynomials, (Academic Press, Inc.).

J. E. A. Dunnage (1966), 'The number of real zeros of a random trigonometric polynomial', Proc. London Math. Soc. 3, 53-84.

J. W. Hamblen (1956), 'Distribution of the roots of quadratic equations with random coeffcients', Ann. Math. Statist. 27, 1136-1143.

I. A. Ibragimov (1962), 'Some limit theorems for stationary processes', Theor. Probab. Appl. 7, 349-382.

I. A. Ibragimov and N. B. Naslova (1971a), 'On the expected number of real zeros of random polynomials (I). Coefficients with zero means', Theor. Probab. Appl. 16, 228-248.

I. A. Ibragimov and N. B. Maslova (1971b), 'On the expected number of real zeros of random polynomials (II). Coefficients with nonzero means', Theor. Probab. Appl. 17, 485-493.

M. Kac (1948), 'On the average number of real roots of a random algebraic equation (II)', Proc. London Math. Soc. 50, 390-408.

N. Renganathan and M. Sambandham (1984), 'On the average number of real zeros of a random trigonometric polynomial with dependent coefficients-(II)', Indian J. Pure Appl. Math. 15(9), 1951-1956.

M. Sambandham (1976a), 'Real zeros of a random polynomial with hyperbolic elements', Indian J. Pure Appl. Math. T(5), 553-556.

M. Sambandham (1976b), 'On random trigonometric polynomials', Indian J. Pure Appl. Math. $7(8), 841-849$.

M. Sambandham (1977), 'On a random algebraic equation', J. Indian Math. Soc. (N.S.) 41 (1-2) 83-97.

M. Sambandham (1979), 'On the average number of real zeros of a class of random algebraic curves', Pacific J. Math. 81, 207-215.

M. Sambandham and C. Maruthachalam (1978), 'On the number of real zeros of a random trigonometric polynomial', J. Indian Math. Soc. (N.S.) 42, (1-4), 143-156. 
M. Shenker (1981), 'The mean number of real roots for one class of random polynomials', Ann. Probab. 9(3), 510-512.

D. C. Stevens (1969), 'The average number of real zeros of a random polynomial', Comm. Pure Appl. Math. 22, 457-477.

E. C. Titchmarsh (1939), The theory of functions, second edition (Oxford University Press).

Ken-Ichi Yoshihara (1978), 'Moment inequalities for mixing sequences', Kodai Math. J. 1 (2), 316-318.

A. Zygmund (1959), Trigonometric series, vol. 1 (Oxford University Press).

Department of Civil Engineering University of Newcastle upon Tyne

Newcastle upon Tyne NE1 7RU

England 